\title{
Tratamento clínico da hemorragia pós-parto: uma revisão integrativa
}

\author{
Clinical management of postpartum hemorrhage: an integrative review \\ Manejo clínico de la hemorragia posparto: una revisión integradora
}

\author{
Antonio Paulo Nunes da Silva \\ ORCID: https://orcid.org/0000-0003-1925-015X \\ Universidade de Pernambuco, Brasil \\ E-mail: paulonunes14081996@gmail.com \\ Maria Eduarda da Silva Dias \\ ORCID: https://orcid.org/0000-0003-3070-209X \\ Universidade de Pernambuco, Brasil \\ E-mail: eduardadias193@gmail.com \\ Pollyana Rodrigues Diniz \\ ORCID: https://orcid.org/0000-0003-2419-3401 \\ Universidade de Pernambuco, Brasil \\ E-mail: pollyrodrigues386@gmail.com \\ Valda Lúcia Moreira Luna \\ ORCID: https://orcid.org/0000-0002-1810-7565 \\ Universidade de Pernambuco, Brasil \\ E-mail: valda.moreira@upe.br \\ George Alessandro Maranhão Conrado \\ ORCID: https://orcid.org/0000-0001-6649-577X \\ Universidade de Pernambuco, Brasil \\ E-mail: george.maranhao@upe.br \\ Pauliana Valéria Machado Galvão \\ ORCID: https://orcid.org/0000-0002-4418-218X \\ Universidade de Pernambuco, Brasil \\ E-mail: pauliana.galvao@upe.br
}

\begin{abstract}
Resumo
Introdução: A Hemorragia Pós-Parto é a causa de aproximadamente $25 \%$ de todas as mortes de gestantes no mundo e é responsável por grande parte das morbidades maternas graves. Assim, objetivou-se avaliar e sintetizar as últimas publicações acerca do tratamento clínico para este evento. Metodologia: Trata-se de uma revisão integrativa realizada no período de janeiro a junho do ano de 2021, que objetivou responder ao seguinte questionamento norteador: "Quais são as evidências recentes disponíveis na literatura acerca do tratamento clínico da hemorragia pós-parto?”. A busca considerou os últimos 5 anos e resultou em 150 artigos, dos quais 12 foram selecionados devido aos critérios de elegibilidade. Resultados: Todos os artigos foram ensaios clínicos randomizados e redigidos em língua inglesa, sendo publicados em periódicos internacionais. Em relação aos países de realização, 4 estudos foram realizados em dois ou mais países e os demais foram executados em 7 países distintos. Discussão: A infusão de ocitocina é considerada o padrão-ouro para o tratamento da hemorragia pós-parto. Contudo, quando há indisponibilidade ou contraindicação ao uso desse medicamento, faltam dados sobre as recomendações para as terapias de segunda linha. Nesse sentido, outros medicamentos como metilergometrina, ácido tranexâmico, misoprostol, entre outros, podem se tornar vantajosos. Conclusão: Devido ao impacto que esta hemorragia tem na saúde materna, é evidente a importância da identificação de estratégias secundárias para seu controle, demonstrando as vias de administração adequadas, segurança e efetividade dos medicamentos.
\end{abstract}

Palavras-chave: Hemorragia; Hemorragia pós-parto; Tratamento.

\begin{abstract}
Introduction: Postpartum hemorrhage is the cause of approximately $25 \%$ of all deaths of pregnant women worldwide and is responsible for a large proportion of severe maternal morbidities. Thus, we aimed to evaluate and synthesize the latest publications on the clinical treatment of this event. Methodology: This is an integrative review conducted from January to June 2021, which aimed to answer the following guiding question: "What is the recent evidence available in the literature on the clinical treatment of postpartum hemorrhage? The search considered the last 5 years and resulted in 150 articles, of which 12 were selected due to eligibility criteria. Results: All articles were randomized clinical trials and written in English, published in international journals. Regarding the countries of performance, 4 studies were performed in two or more countries and the others were performed in 7 different countries. Discussion: Oxytocin infusion is considered the gold standard for the treatment of postpartum hemorrhage. However, when there is unavailability or contraindication to the use of this drug, data on recommendations for second-line therapies are
\end{abstract}


lacking. In this sense, other drugs such as methylergometrine, tranexamic acid, misoprostol, among others, may become advantageous. Conclusion: Due to the impact that this hemorrhage has on maternal health, the importance of identifying secondary strategies for its control is evident, demonstrating the appropriate routes of administration, safety and effectiveness of the drugs.

Keywords: Hemorrhage; Postpartum hemorrhage; Treatment.

\section{Resumen}

Introducción: La hemorragia postparto es la causa de aproximadamente el $25 \%$ de todas las muertes de gestantes en el mundo y es responsable de gran parte de la morbilidad materna grave. Por lo tanto, el objetivo era evaluar y sintetizar las últimas publicaciones sobre el tratamiento clínico para este evento. Metodología: Se trata de una revisión integradora realizada en el período de enero a junio del año 2021, que tenía como objetivo responder a la siguiente pregunta norteamericana: "¿Cuáles son las evidencias recientes disponibles en la literatura sobre el tratamiento clínico de la hemorragia postparto?". La búsqueda tuvo en cuenta los últimos 5 años y dio como resultado 150 artículos, de los cuales se seleccionaron 12 debido a los criterios de elegibilidad. Resultados: Todos los artículos fueron ensayos clínicos aleatorios y rediseñados en lengua inglesa, siendo publicados en periódicos internacionales. En cuanto a los países de realización, 4 estudios se realizaron en dos o más países y los demás se realizaron en 7 países diferentes. Discusión: La infusión de oxitocina se considera el estándar de oro para el tratamiento de la hemorragia posparto. Sin embargo, cuando no hay disponibilidad o contraindicación para el uso de este fármaco, faltan datos sobre las recomendaciones para las terapias de segunda línea. En este sentido, otros fármacos como la metilergometrina, el ácido tranexámico, el misoprostol, entre otros, pueden resultar ventajosos. Conclusión: Debido al impacto que esta hemorragia tiene en la salud materna, es evidente la importancia de la identificación de estrategias secundarias para su control, demostrando las vías de administración adecuadas, la seguridad y la eficacia de los medicamentos.

Palabras clave: Hemorragia; Hemorragia posparto; Tratamiento.

\section{Introdução}

O pós-parto ou puerpério compreende um período na vida de uma mulher no qual ocorrem manifestações involutivas e recuperativas do organismo materno após o parto, em que os profissionais de saúde devem fornecer informações e realizar avaliações específicas, uma vez que está sujeito a complicações. Divide-se em puerpério imediato $\left(1^{\circ}\right.$ ao $10^{\circ}$ dia pós-parto), tardio ( $11^{\circ}$ ao $45^{\circ}$ dia) e remoto (após o $45^{\circ}$ dia) (Baratieri; Natal, 2019).

Um dos principais problemas deste intervalo é a hemorragia pós-parto (HPP), definida como a perda sanguínea acima de $500 \mathrm{~mL}$ após parto vaginal ou acima de $1.000 \mathrm{~mL}$ após cesariana nas primeiras 24 horas ou, ainda, qualquer perda de sangue pelo trato genital capaz de causar instabilidade hemodinâmica (Alves et al., 2020; OPAS, 2018).

Dentro deste conceito, existe uma subdivisão, denominada HPP maciça, que considera o sangramento nas primeiras 24 horas após o parto (por qualquer via) superior a $2.000 \mathrm{~mL}$ ou que necessite da transfusão mínima de $1.200 \mathrm{~mL}$ (4 unidades) de concentrado de hemácias ou que resulte na queda de hemoglobina $\geq 4 \mathrm{~g} / \mathrm{dL}$ ou em distúrbio de coagulação (OPAS, 2018).

A HPP afeta aproximadamente $2,0 \%$ a $7,0 \%$ de todas as mulheres no período puerperal e é a causa de aproximadamente $25 \%$ de todas as mortes de gestantes no mundo, principalmente em países de baixa e média renda, com cerca de 140.000 mortes anuais e frequência de uma morte a cada quatro minutos. Em sua maioria, essas mortes são consideradas evitáveis, denunciando a precariedade da assistência à saúde em nações mais pobres (Alves et al., 2020; Andrade et al., 2019; Dumont et al., 2017; Koch \& Rattmann, 2019).

Além disso, esta intercorrência é responsável por grande parte das morbidades maternas graves, como internações hospitalares prolongadas, necessidade de transfusões sanguíneas, inclusive com evolução para choque e disfunção orgânica, e procedimentos cirúrgicos que podem levar à perda da função reprodutiva, sequelas físicas e/ou emocionais (Alves et al., 2020; Andrade et al., 2019; Koch \& Rattmann, 2019).

No Brasil, as hemorragias constituem a segunda causa de morte materna, com a HPP correspondendo a 40,8\% do total das hemorragias obstétricas. Desse percentual, grande parte das mortes pode ser evitada através do uso de ocitócitos de forma profilática durante a terceira fase do parto e do manejo imediato e adequado da hemorragia. Ainda assim, acredita-se que 
aproximadamente $6,0 \%$ de todos os partos evoluam para a HPP e 1,9\% para a HPP grave ( $\geq 1.000 \mathrm{~mL}$ de perda sanguínea) (Alves et al., 2020; Koch; Rattmann, 2019).

A maioria das causas pode ser agrupada no mnemônico "4 Ts": "tônus" (79,0\%), que inclui a atonia uterina; "trauma" (19,0\%), que agrupa lacerações, hematomas, inversão e rotura uterina; "tecido" $(10,0 \%)$, abrangendo retenção de tecido placentário, coágulos e acretismo placentário e, por fim, "trombina" (1,0\%), compondo as coagulopatias congênitas ou adquiridas e o uso de medicamentos anticoagulantes (OPAS, 2018).

Outros fatores de risco também se destacam, como anemia, síndromes hipertensivas, placenta prévia ou de inserção baixa, hematócrito menor que 30,0\%, plaquetas abaixo de $100.000 / \mathrm{mm}^{3}$, sangramento ativo à admissão e descolamento prematuro da placenta (Alves et al., 2020). Diante desse cenário, objetivou-se avaliar e sintetizar as últimas publicações acerca do tratamento clínico para a HPP, tendo em vista a deteç̧ão, o diagnóstico e, sobretudo, o manejo clínico precoce.

\section{Metodologia}

O presente estudo trata-se de uma revisão integrativa operacionalizada por meio das seguintes etapas: (1) formulação de questão de pesquisa; (2) estabelecimento de critérios de inclusão e exclusão; (3) coleta de dados; (4) avaliação dos estudos selecionados; (5) análise e interpretação dos resultados; e (6) apresentação da súmula do conhecimento.

Nesse sentido, esta pesquisa objetivou responder ao seguinte questionamento norteador: "Quais são as evidências recentes disponíveis na literatura acerca do tratamento clínico da HPP?”. Assim, no período de janeiro a junho do ano de 2021, foram pesquisadas publicações científicas indexadas, empregando a base de dados Medical Literature Analysis and Retrievel System Online (Medline). Para tanto, foram adotados os Descritores em Ciências da Saúde (DECS) controlados: "Hemorrhage", "Postpartum", "Clinical treatment", "Oxytocin", "Blood coagulation factor", integrados pelo operador booleano AND.

Foram estabelecidos como critérios para a seleção: estudos clínicos randomizados, publicados entre 2016 e 2021, disponibilizados integralmente, no idioma inglês, que abordassem a temática pesquisada. Não foram incluídos os estudos do tipo revisão sistemática e metanálise, uma vez que realizam, respectivamente, a descrição/método estatístico de análise de uma evidência já sumarizada/reunida sistematicamente. Foram excluídos os estudos que não respondiam à questão norteadora e os que se repetiram na base de dados.

A busca inicial na literatura resultou em uma coleção de 150 publicações. Após esta etapa, ocorreu a leitura minuciosa dos títulos e dos resumos dos estudos, na qual foram excluídos 120 artigos por não se enquadrarem na temática abordada e 5 artigos que se repetiram na base de dados. Assim, à luz dos critérios de elegibilidade, 25 artigos foram pré-selecionados. Posteriormente, realizou-se a leitura na íntegra dos artigos, dos quais 12 foram incluídos por responderem satisfatoriamente à questão norteadora e, portanto, compuseram o conjunto final de análise desta revisão. O fluxograma com o detalhamento das etapas de seleção das publicações é apresentado na Figura 1.

Com vistas a assegurar os aspectos éticos inerentes a essa pesquisa, a autoria e as citações de cada publicação foram devidamente respeitadas por meio de sua referenciação. Cabe salientar ainda que, por se tratar de um estudo de revisão de literatura e, por conseguinte, sem o envolvimento de seres humanos, é dispensada a apreciação pelo Comitê de Ética em Pesquisa segundo a Resolução 510/2016 do Conselho Nacional de Saúde. 
Figura 1. Fluxograma do processo de seleção dos estudos incluídos na revisão.

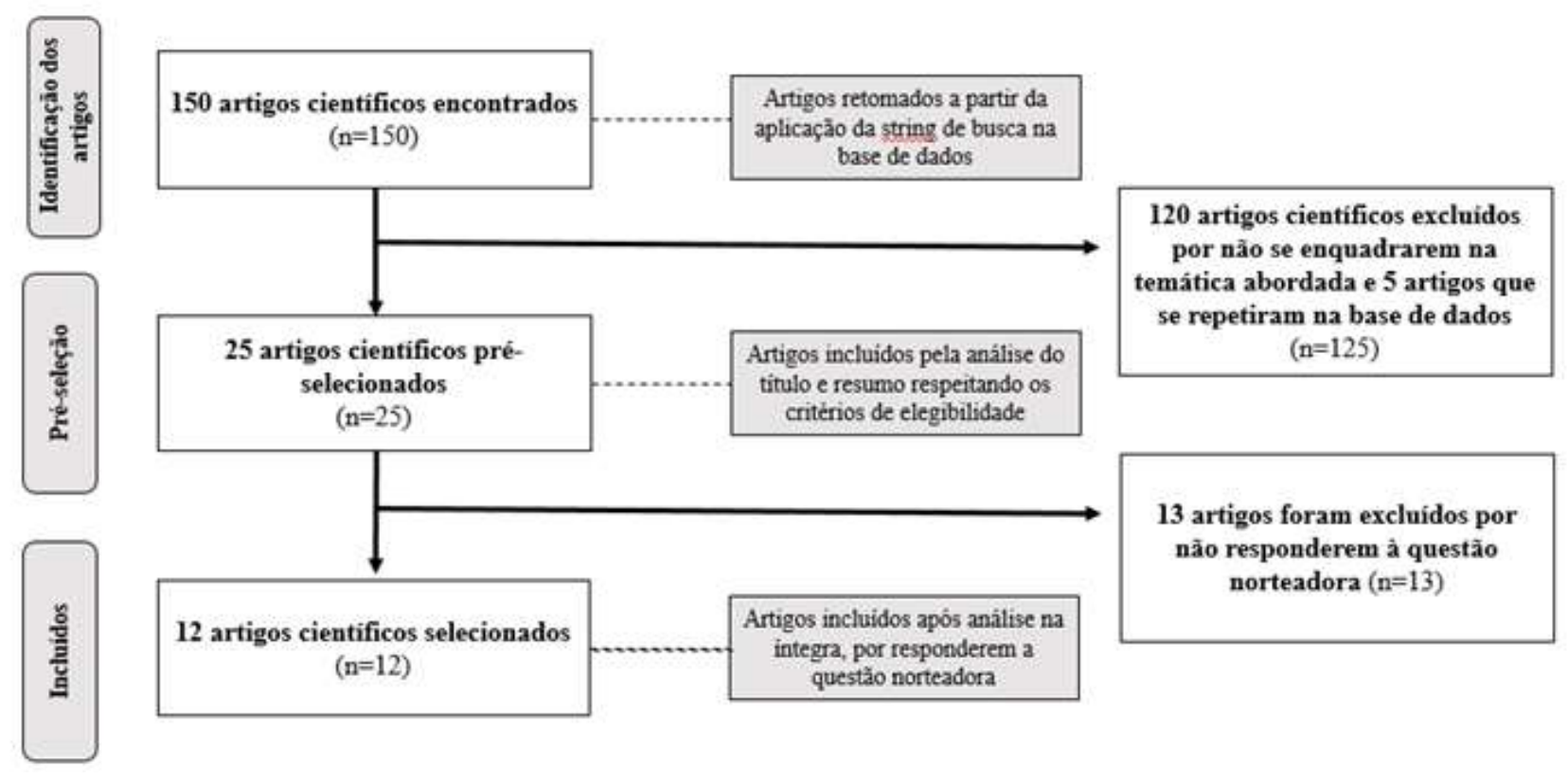

Fonte: Pesquisa própria (2021).

\section{Resultados}

Os artigos selecionados e analisados estão apresentados na Tabela 1, logo abaixo, a fim de facilitar a visualização e a compreensão dos dados.

No que concerne ao ano de publicação, 1 artigo foi publicado em 2021 (8,3\%); 2 artigos em 2019 (16,7\%); 5 artigos em 2018 (41,7\%) e 4 artigos foram publicados em 2017 (33,3\%), totalizando 12 estudos. Referente à localização geográfica onde os estudos foram realizados, 4 eram provenientes de dois ou mais países e o restante foi realizado no Egito (2), Dinamarca (1), Irã (1), França (1), Argentina (1), Índia (1) e Suíça (1). 
Research, Society and Development, v. 10, n. 16, e84101623363, 2021

(CC BY 4.0) | ISSN 2525-3409 | DOI: http://dx.doi.org/10.33448/rsd-v10i16.23363

Tabela 1. Distribuição dos artigos incluídos na revisão segundo autores/ano, objetivo, método e conclusões.

Autor/ano Objetivo Principais resultados Conclusões

Objetivo

Principais resultados

Conclusões

Brenner et al. Examinar os efeitos do ácido tranexâmico Entre 14.923 mulheres randomizadas dentro de $3 \mathrm{~h}$ após o parto, houve 216 mortes e 383

(2018) (AT) sobre morte e histerectomia devido a sangramento, realizando análises repetidas.

histerectomias devido a HPP. Com o uso de AT, houve redução significativa no risco

O AT reduz o risco de morte por morte devido ao sangramento, o mesmo não ocorrendo quanto ao risco de histerectomia.

HPP mas é incerto se reduz apresentam

histerectomia por sangramento.

Cecilia et al. Comparar a eficácia da ocitocina intravenosa Foram incluídas 271 pacientes. 135 receberam dose única (10 unidades) e 136 fizeram a (2018) em dose única por 2-4h (total = 10 unidades) com infusão de manutenção de ocitocina po $8-12 \mathrm{~h}$ (total $=30$ unidades) em mulheres no pós-operatorio de parto cesáreo pro prevenção de HPP.

infusão de manutenção (30 unidades). Ambos os grupos eram comparáveis em relação às características demográficas. Quando analisados, verificou-se que ambos os regimes foram gualmente eficazes na prevenção da HPP em mulheres no pós-operatório, sendo associados a quantidade semelhante de perda de sangue durante o período operatório e pósoperatório.

Não houve diferença em qualquer uma das medidas de resultados primários ou secundários nos dois grupos. Portanto, o regime de baixa dose de ocitocina é tão eficaz quanto o regime de alta dose na prevenção da HPP em mulheres no pósoperatório de parto cesáreo.

Colucci et al. Avaliar o protocolo de tratamento Foram comparadas pacientes previamente tratadas com fator VII ativado recombinante (2018) padronizado em pacientes com HPP severa (rFVIIa) em sangramento maciço (20 pacientes), pacientes tratadas sem orientação padronizado em pacientes com $\mathrm{HPP}$ severa (rFVIla) em sangramento maciço ( 20 pacientes), pacientes tratadas sem orientação tratadas antes da adoção desta estratégia.

coorte do estudo, a duração do sangramento foi menor do que em ambas as coortes históricas 1 e 2 . O número de hemoderivados transfundidos antes da administração de agentes hemostáticos (fibrinogênio e / ou rFVIIa, se necessário) foi significativamente menor na coorte do estudo. A quantidade de hemoderivados transfundidos após a administração de fibrinogênio e / ou rFVIIa não foi diferente entre os 3 grupos.

Ducloy- Avaliar os benefícios e a segurança do

Bouthors et al. concentrado de fibrinogênio humano precoce

(2021) no tratamento da HPP.

Foram incluídos 437 pacientes. 224 receberam concentrado de fibrinogênio (CF, grupo 1) e 213 placebo (grupo 2). A perda de sangue e fibrinogênio plasmático foram semelhantes em ambos os grupos, com taxas de falha de $40,0 \%$ e $42,4 \%$ respectivamente. Não foram observadas diferenças significativas nos resultados de eficácia secundários. O fibrinogênio plasmático médio permaneceu inalterado no grupo 1 e diminuiu $0,56 \mathrm{~g} / 1$ no grupo 2 . Nenhum efeito tromboembólico ou outros efeitos adversos relevantes foram relatados no grupo 1.

AT = ácido tranexâmico; HPP = hemorragia pós-parto; $\mathrm{h}=$ hora; $\mathrm{rFVIIa}=$ fator VII ativado recombinante; $\mathrm{CF}=$ concentrado de fibrinogênio; $\mathrm{g} / \mathrm{l}=$ grama por litro; $\mathrm{g}=$ grama; continua... 
Research, Society and Development, v. 10, n. 16, e84101623363, 2021

(CC BY 4.0) | ISSN 2525-3409 | DOI: http://dx.doi.org/10.33448/rsd-v10i16.23363

Tabela 1. Distribuição dos artigos incluídos na revisão segundo autores/ano, objetivo, método e conclusões. (continuação)

\begin{tabular}{|c|c|c|c|}
\hline Autor/ano & Objetivo & Principais resultados & Conclusões \\
\hline $\begin{array}{l}\text { Dumont et } \\
\text { al. (2017) }\end{array}$ & $\begin{array}{l}\text { Avaliar a eficácia do tamponamento uterino de } \\
\text { baixo custo como adjuvante do misoprostol } \\
\text { para o tratamento da HPP não controlada em } \\
\text { ambientes com poucos recursos. }\end{array}$ & $\begin{array}{l}\text { Foram comparados os resultados de } 116 \text { mulheres com HPP não controlada. } 57 \text { pacientes } \\
\text { usaram misoprostol e tamponamento e } 59 \text { usaram apenas misoprostol. A proporção de } \\
\text { mulheres com cirurgia invasiva ou que morreram antes da alta hospitalar não diferiu } \\
\text { significativamente entre o grupo de intervenção e o grupo de controle. Uma proporção } \\
\text { maior de mulheres com tamponamento e misoprostol versus misoprostol sozinho perdeu } \\
\text { mais de } 1000 \mathrm{ml} \text { de sangue. A taxa de letalidade foi maior no grupo de tamponamento do } \\
\text { que no grupo controle. }\end{array}$ & $\begin{array}{l}\text { Não há benefício com o tamponamento para o } \\
\text { tratamento da atonia uterina. O uso de } \\
\text { tamponamento uterino de baixo custo não tem } \\
\text { efeito significativo no recurso à cirurgia } \\
\text { invasiva. No entanto, atrasos no manejo da HPP } \\
\text { podem explicar o aumento da morbidade e } \\
\text { mortalidade materna entre as mulheres tratadas } \\
\text { com tamponamento e misoprostol. }\end{array}$ \\
\hline $\begin{array}{l}\text { Durocher } \\
\text { et al. } \\
(2019)\end{array}$ & $\begin{array}{l}\text { Avaliar o impacto da infusão de ocitocina } \\
\text { intravenosa contra a intramuscular na perda de } \\
\text { sangue pós-parto e nas taxas de HPP quando } \\
\text { administrada durante o terceiro estágio do } \\
\text { trabalho de parto. }\end{array}$ & $\begin{array}{l}\text { Foram inscritas } 239 \text { (infusão intravenosa - IV) e } 241 \text { (injeção intramuscular - IM) mulheres } \\
\text { com características basais comparáveis. A perda média de sangue foi } 43 \mathrm{ml} \text { a menos no } \\
\text { grupo de infusão IV. Taxas de perda de sangue } \geq 500 \mathrm{ml} \text { foram semelhantes. As mulheres } \\
\text { no grupo de infusão IV receberam significativamente menos uterotônicos adicionais do } \\
\text { que as mulheres no grupo IM. Mulheres com HPP no grupo IM experimentaram um } \\
\text { aumento maior no índice de choque após o parto, o que pode ter influenciado o recurso a } \\
\text { intervenções adicionais. }\end{array}$ & $\begin{array}{l}\text { A via de administração de ocitocina para a } \\
\text { prevenção da HPP não teve impacto } \\
\text { significativo na perda de sangue após o parto } \\
\text { vaginal. No entanto, diferenças foram } \\
\text { observadas entre uterotônicos adicionais, } \\
\text { favorecendo a infusão IV sobre a IM. Em } \\
\text { ambientes onde os acessos intravenosos são } \\
\text { colocados, a infusão de ocitocina pode ser } \\
\text { preferível à injeção IM. }\end{array}$ \\
\hline $\begin{array}{l}\text { Holm et } \\
\text { al. }(2017)\end{array}$ & $\begin{array}{l}\text { Avaliar a viabilidade e os resultados } \\
\text { exploratórios de uma alta infusão de dose } \\
\text { única e elevada de isomaltosídeo de ferro em } \\
\text { comparação com a transfusão de hemácias } \\
\text { para o tratamento de anemia pós-parto grave } \\
\text { em mulheres estáveis. }\end{array}$ & $\begin{array}{l}\text { Foram rastreadas } 162 \text { mulheres e incluídas } 13(8,0 \%) \text {. A transfusão de hemácias foi } \\
\text { associada a uma maior hemoglobina }(\mathrm{Hb}) \text { no dia } 1 \text {, inibição da reticulocitose durante a } \\
\text { primeira semana e baixos níveis de ferro. O ferro intravenoso foi associado a reticulocitose } \\
\text { aumentada durante a primeira semana, estoques de ferro repleto e uma Hb mais alta nas } \\
\text { semanas 3-12. }\end{array}$ & $\begin{array}{l}\text { Os resultados preliminares sugeriram que uma } \\
\text { infusão de dose única de isomaltosídeo de ferro } \\
\text { pode ser uma alternativa atraente para a } \\
\text { transfusão de hemácias no tratamento da } \\
\text { anemia pós-parto grave. }\end{array}$ \\
\hline $\begin{array}{l}\mathrm{Li} \text { et al. } \\
(2018)\end{array}$ & \begin{tabular}{lllll} 
Avaliar a & relação & \multicolumn{2}{l}{ custo-eficácia } & da \\
administração precoce & de AT para & AT \\
tratamento de HPP. & & &
\end{tabular} & $\begin{array}{l}\text { O tratamento precoce da HPP com AT gerou um ganho médio de } 0,18 \text { anos de vida } \\
\text { ajustado para qualidade (QALYs) a um custo adicional de US } \$ 37,12 \text { por paciente na } \\
\text { Nigéria e um ganho médio de } 0,08 \text { QALYs a um custo adicional de US\$ } 6,55 \text { por paciente } \\
\text { no Paquistão. As razões de custo-efetividade incrementais estavam abaixo do limite } \\
\text { inferior da faixa do limite de custo-efetividade. }\end{array}$ & $\begin{array}{l}\text { O tratamento precoce de HPP com AT é custo- } \\
\text { efetivo em ambos os países. }\end{array}$ \\
\hline
\end{tabular}

HPP = hemorragia pós-parto; $\mathrm{CF}$ = concentrado de fibrinogênio; $\mathrm{g} / \mathrm{l}$ = grama por litro; IM = injeção intramuscular; IV = infusão intravenosa; continua... 
Tabela 1. Distribuição dos artigos incluídos na revisão segundo autores/ano, objetivo, método e conclusões. (Continuação).

\section{Autor/ano Objetivo}

Shady et al. Avaliar o efeito do AT oral (2019) profilático mais misoprostol
sublingual sobre a quantidade de perda sanguínea após o parto vaginal em mulheres com baixo risco de HPP.

Sweed et al. Comparar a eficácia da As pacientes foram alocadas em 3 grupos de 151 pacientes que usaram doses (2019) administração de diferentes doses de misoprostol retal antes da cesariana para reduzir a perda sanguínea intra e pós-operatória.

\section{Principais resultados}

\section{Conclusões} sublingual $(600 \mu \mathrm{g})$. Houve um nível de $\mathrm{Hb}$ inferior estatisticamente significativo e

WOMAN Avaliar os efeitos da administração Foram inscritas 20.060 mulheres e designadas aleatoriamente para receber AT (1g $\begin{array}{ll}\text { Trial } & \text { precoce de AT na morte, } \\ \text { Collaborators } & \text { histerectomia e outros resultados }\end{array}$ (2017) relevantes em mulheres com HPP. (2018) Comparar o efeito do AT intravenoso e análogo prostaglandina na redução da HPP resultante da atonia uterina em mulheres submetidas à cesariana ou ao parto vaginal.
As pacientes foram divididas em 3 grupos de 120 pacientes. O grupo 1 foi submetido à infusão de ocitocina intravenosa (10 unidades), o grupo 2 usou misoprostol sublingual $(600 \mu \mathrm{g})$ e o grupo 3 usou AT oral $(1000 \mathrm{mg})$ e misoprostol maior perda de sangue no grupo 2. Houve um maior nível de Hb estatisticamente significativo e menor perda de sangue no grupo 3 em comparação com o grupo 1 . HPP ocorreu em 16,7\% das mulheres no grupo 2 em comparação a 1,7\% no grupo 1 e nenhum caso de HPP no grupo 3. diferentes de misoprostol retal (grupo 1: $200 \mu \mathrm{g}$; grupo 2: 400 $\mu \mathrm{g}$; grupo 3: $600 \mu \mathrm{g}$. A perda sanguínea intra-operatória foi maior em pacientes do grupo 1 do que os demais grupos, mas não foi encontrada diferença estatística entre os grupos 2 e 3 . A incidência de efeitos colaterais como febre e calafrios aumenta com o aumento da dose de misoprostol.

Em locais como área rural ou parto em domicílio, nos quais a ocitocina não está disponível, a alternativa AT oral mais misoprostol sublingual pode ser considerada uma linha eficaz na prevenção da HPP.

A administração retal de misoprostol para a prevenção da HPP e diminuição da perda sanguínea intra-operatória durante a cesariana é uma boa alternativa aos outros uterotônicos. A melhor dose precisa de mais pesquisas para ser acordada.

O AT reduz a morte devido ao sangramento em mulheres com HPP sem efeitos adversos. Quando usado como tratamento para HPP, o AT deve ser administrado o mais rápido possível após o início do sangramento. AT. Os eventos adversos, incluindo eventos tromboembólicos, não diferiram significativamente nos dois grupos.

A administração intravenosa de AT obteve efeitos comparáveis ao análogo da prostaglandina na redução da HPP em mulheres com atonia uterina e naquelas submetidas à cesariana ou ao parto vaginal.

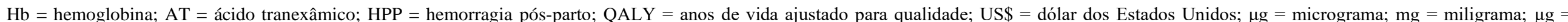
micrograma; AT = ácido tranexâmico; AT = ácido tranexâmico; HPP = hemorragia pós-parto; g = grama; IV = intra venoso; IM = intra muscular. Fonte: Pesquisa própria (2021). 


\section{Discussão}

\section{Ocitocina}

A administração de ocitocina durante a terceira fase do trabalho de parto é amplamente defendida para auxiliar nas contrações uterinas e reduzir o risco de sangramento. Ademais, o uso profilático de rotina reduz a incidência de HPP em aproximadamente $40 \%$, resultando na diminuição da morbidade materna. No entanto, ainda não há consenso em relação à dose e velocidade de administração da droga (Cecilia et al., 2018; Durocher et al., 2019).

Embora doses maiores possam ser mais eficazes na prevenção da atonia uterina, estão associadas a efeitos colaterais significativos, incluindo hipotensão, náusea, vômito, dor torácica, cefaléia, rubor, isquemia miocárdica, alterações do segmento ST-T, edema pulmonar e até convulsões, sobretudo em doses administradas rapidamente, apresentando efeito cumulativo (Cecilia et al., 2018).

A injeção intramuscular é a via mais difundida devido ao seu perfil de segurança favorável e às vantagens práticas em relação à infusão intravenosa, principalmente em locais com poucos recursos. De forma profilática, não houve diferença significativa entre o uso de ocitocina IV ou IM em relação à perda sanguínea ou à incidência de HPP. Por outro lado, houve redução clínica e estatisticamente relevante no uso de uterotônicos adicionais. Assim, se um acesso intravenoso já estiver instalado antes do parto, a profilaxia IV seria mais fácil e poderia reduzir a necessidade subsequente de uterotônicos complementares. Em contrapartida, se não houver um acesso estabelecido, a via IM provavelmente é a maneira mais eficiente de administração (Durocher et al., 2019).

No parto cesáreo, um regime de baixa dose de ocitocina foi considerado tão eficaz quanto o de alta dose na prevenção da HPP, uma vez que, se um número ideal de receptores de ocitocina já estiver ocupado, maior incremento na dose só aumentará a incidência e a gravidade dos efeitos colaterais sem qualquer benefício na contração uterina. Doses em bolus de 0,5-3,0 UI estão associadas a contrações uterinas adequadas e o aumento para mais de 5,0 UI eleva a incidência de hipotensão sem quaisquer benefícios na contratilidade muscular. Ainda, descobriu-se in vitro e in vivo que o uso contínuo de ocitocina por aproximadamente 4,2 horas pode resultar na dessensibilização do receptor uterino da ocitocina, dificultando o trabalho de parto (Cecilia et al., 2018).

\section{Ácido tranexâmico (AT)}

O AT é um dos principais agentes antifibrinolíticos que têm sido utilizados em várias cirurgias. Dessa forma, pesquisas sobre tais agentes em hemorragias significativas mostraram que a administração de AT reduz substancialmente a taxa de mortalidade na população de pacientes com sangramento por trauma. Além disso, diversos estudos sugeriram que a administração de AT reduz a perda sanguínea perioperatória e a incidência de HPP em pacientes submetidas a parto cesáreo ou vaginal eletivo (Zargar et al., 2018).

Nesse contexto, o AT é recomendado para o tratamento da hemorragia pós-parto primária se a ocitocina e outros uterotônicos falharam em controlar o sangramento ou se houver suspeita de que o sangramento pode ser parcialmente devido ao trauma. Sendo assim, é um medicamento que bloqueia o local de ligação da lisina do plasminogênio à fibrina. Consequentemente, o coágulo não se decompõe, a fibrinólise é inibida e o sangramento excessivo é reduzido (Brenner et al., 2018; Shady et al., 2019).

Mulheres com 16 anos ou mais com diagnóstico clínico de HPP após parto vaginal ou cesariana de 193 hospitais em 21 países foram recrutadas para participar de um estudo. Essas mulheres foram aleatoriamente designadas para receber $1 \mathrm{~g}$ de AT intravenoso ou placebo. Os resultados sugerem que, se o AT for usado no tratamento da HPP, sua administração de forma precoce é mais eficaz. Dessa forma, o tratamento dentro de 3 horas após o nascimento reduziu significativamente a necessidade de laparotomia e a ocorrência de óbito devido ao sangramento (WOMAN Trial Collaborators, 2017). 
A administração do AT ocorre principalmente através da injeção intravenosa. A concentração plasmática efetiva de AT que demonstrou causar inibição significativa da fibrinólise sistêmica em adultos foi de 5,0 a 10,0 $\mu \mathrm{g} / \mathrm{mL}$, com inibição quase máxima entre 10,0 e 15,0 $\mu \mathrm{g} / \mathrm{mL}$, e o tempo de meia-vida foi de 2 a 3 horas. Embora não seja comparável à administração intravenosa de AT, onde a concentração plasmática efetiva é alcançada imediatamente, o AT administrado por via oral mostra potencial para ser eficaz no tratamento de HPP (Brenner et al., 2018).

A relação custo-efetividade da adição de AT ao tratamento usual para mulheres com HPP mostrou que esta estratégia gerou um aumento no QALYs a um baixo custo adicional na Nigéria e no Paquistão. Sendo assim, como uma intervenção barata e potencialmente salvadora, o AT se apresentou eficaz em termos de custos em diferentes ambientes (Li et al., 2018).

\section{Misoprostol}

Em ambientes de poucos recursos, o misoprostol, análogo da prostaglandina E1, torna-se vantajoso, embora ainda possua baixa evidência de sua eficácia. Isto porque é fácil de armazenar em temperatura ambiente, de baixo custo, relativamente seguro e absorvido por várias vias: vaginal, retal, oral ou sublingual (Dumont et al., 2017).

Além do uso em âmbito hospitalar, é útil em situações de partos realizados por parteiras tradicionais, em partos domiciliares sem a assistência profissional e onde as mulheres estão em maior risco de HPP grave (Shady et al., 2019). Quanto à dosagem de administração, o uso de uma dose de $400 \mu \mathrm{g}$ de misoprostol retal ou mais traz melhor benefício do que $200 \mu \mathrm{g}$ para a redução da perda sanguínea intra e pós-operatória relacionada à cesariana (Sweed et al., 2019).

Nessa perspectiva, o misoprostol foi comparado à ergometrina, ocitocina, mostrando resultados comparáveis. Sua administração retal mostrou taxas de absorção mais lentas e níveis de pico mais baixos quando comparados às vias sublingual e oral, mas também mostrou redução na incidência de efeitos adversos (Sweed et al., 2019). Ademais, sabe-se que o uso profilático da associação entre AT e misoprostol durante o parto vaginal reduz efetivamente a perda de sangue pós-parto, as necessidades de transfusão de sangue e também diminuem a incidência de HPP comparativamente ao uso do misoprostol isoladamente (Shady et al., 2019).

\section{Plasma/transfusão}

A coagulopatia é um dos principais agravantes da perda de sangue. Durante a HPP, o fibrinogênio plasmático é o primeiro fator de coagulação a cair precipitadamente devido ao sangramento, hiperfibrinólise e hemodiluição. Além disso, o fibrinogênio é um biomarcador precoce para o agravamento da HPP. O risco de progressão para HPP grave aumenta quase três vezes para cada diminuição de $1 \mathrm{~g} / \mathrm{L}$ na concentração de fibrinogênio, e um valor preditivo positivo para progressão para HPP grave é observado com concentrações de fibrinogênio menor que 2g/L (Ducloy-Bouthors et al., 2021).

Nesse contexto, fibrinogênio menor que 3g/L, medido durante o início a HPP, está associado à progressão para hemorragia maciça, transfusão de glóbulos vermelhos e plasma fresco congelado e procedimentos invasivos. Fibrinogênio maior que $4 \mathrm{~g} / \mathrm{L}$ está menos frequentemente associado à progressão do sangramento. Durante a HPP, não se sabe se o fibrinogênio deve ser mantido em um nível que é normal a termo (maior que $4 \mathrm{~g} / \mathrm{L}$ ), acima da faixa de não grávidas (maior que $2 \mathrm{~g} / \mathrm{L}$ ) ou um nível intermediário $(3 \mathrm{~g} / \mathrm{L})$. Portanto, a terapia de reposição de fibrinogênio para sangramento grave ganhou popularidade (Colucci et al., 2018; Ducloy-Bouthors et al., 2021).

Uma força-tarefa multidisciplinar para tratamento avançado de sangramento em pacientes com HPP recomenda a reposição de fibrinogênio se o nível plasmático cair abaixo de $2 \mathrm{~g} / \mathrm{L}$. No entanto, continua a ser debatido se a administração sistemática de fibrinogênio em um estágio anterior pode ser útil, ou seja, antes da determinação do nível de plasma, uma vez que a utilidade clínica do fibrinogênio laboratorial para prever a progressão da HPP apresenta limitações, porque os resultados 
levam de 60 a 90 minutos para serem disponibilizados. Ao mesmo tempo, diversos estudos ainda afirmam que a suplementação de fibrinogênio é eficaz e benéfica (Colucci et al., 2018; Ducloy-Bouthors et al., 2021).

\section{Suplemento de ferro}

Quanto às recomendações para o tratamento da anemia pós-parto moderada a grave, geralmente são utilizados suplementos de ferro intravenoso, sendo este considerado superior aos suplementos orais de ferro em termos de aumento mais rápido da ferritina e hemoglobina séricas, e melhores escores de fadiga materna no período pós-parto. Ainda mais, no que concerne a transfusão sanguínea, está indicada apenas para sintomas graves de anemia e valores de hemoglobina abaixo de $7 \mathrm{~g} / \mathrm{dL}$ (Holm et al., 2017).

\section{Conclusão}

A HPP continua a figurar como uma das principais causas de morbidade e mortalidade materna em todo o mundo. Sendo assim, destaca-se a importância da identificação de estratégias secundárias para seu controle. No que tange às principais recomendações acerca do manejo clínico, a ocitocina é atualmente o medicamento mais amplamente difundido visando auxiliar nas contrações uterinas e reduzir o risco de sangramento. Nesse sentido, não houve diferença em relação à via de administração de ocitocina para a prevenção da HPP após parto normal, mas a via IV promoveu menor necessidade de uterotônicos adicionais. Assim, em locais onde sejam obtidos acessos intravenosos rotineiramente, é preferível optar pela infusão de ocitocina IV de alta qualidade, sendo aceitável a injeção IM quando o acesso venoso não for rotina.

Em locais como área rural ou em situação de parto em domicílio, nos quais a ocitocina não está disponível, a alternativa AT oral mais misoprostol sublingual pode ser considerada uma linha eficaz na prevenção da HPP. O seu tratamento precoce com AT é custo-efetivo, bem como reduz a mortalidade devido ao sangramento em mulheres com hemorragia pósparto sem produzir efeitos adversos. Quando usado como tratamento para hemorragia pós-parto, o AT deve ser administrado o mais rápido possível após o início do sangramento.

Para a prevenção da hemorragia pós-parto e diminuição da perda sanguínea intra-operatória durante a cesariana, a administração de misoprostol é uma boa alternativa aos outros uterotônicos. Ainda assim, a melhor dose a ser usada precisa de mais pesquisas para ser melhor definida. Em relação aos fatores de coagulação, a infusão precoce e sistemática de fibrinogênio de $3 \mathrm{~g}$ na HPP não reduziu a perda de sangue, a necessidade de transfusão ou a anemia pós-parto, porém reduziu a hipofibrinogenemia e foi segura. Por sua vez, a infusão de dose única de isomaltosídeo de ferro pode ser uma alternativa atraente para a transfusão de hemácias no tratamento da anemia pós-parto grave. Diante disso, há muito a esclarecer no que concerne ao tratamento adequado da HPP, sendo necessário a produção de estudos sobre a efetividade da via de administração da ocitocina mediante o parto cesáreo, bem como de pesquisas para determinar a dosagem específica e efetiva para o sucesso da reversão do quadro de HPP com o AT, o misoprostol e o uso combinado destas duas drogas, inclusive considerando diversas possibilidades de vias de administração.

\section{Referências}

Alves, Á. L. L., Francisco, A. A., Osanan, G. C., \& Vieira, L. B. (2020). Hemorragia pós-parto: Prevenção, diagnóstico e manejo não cirúrgico. FEBRASGO Position Statement, 5, 1-7.

Andrade, P. de O. N., Oliveira, S. C. de, Morais, S. C. R. V., Guedes, T. G., Melo, G. P. de, \& Linhares, F. M. P. (2019). Validação de cenário de simulação clínica no manejo da hemorragia pós-parto. Revista Brasileira de Enfermagem, 72(3), 624-631. https://doi.org/10.1590/0034-7167-2018-0065

Baratieri, T., \& Natal, S. (2019). Ações do programa de puerpério na atenção primária: Uma revisão integrativa. Ciência \& Saúde Coletiva, 24(11), 42274238. https://doi.org/10.1590/1413-812320182411.28112017

Brenner, A., Shakur-Still, H., Chaudhri, R., Fawole, B., Arulkumaran, S., \& Roberts, I. (2018). The impact of early outcome events on the effect of tranexamic 
acid in post-partum haemorrhage: An exploratory subgroup analysis of the WOMAN trial. BMC Pregnancy and Childbirth, 18 , 215. https://doi.org/10.1186/s12884-018-1855-5

Campo Grande. (2021). Atenção à puérpera e ao recém-nascido: Orientações técnicas para profissionais 2021. SESAU.

Cecilia, M., Vijayaselvi, R., Bansal, R., Lakshmi, L., \& Jose, R. (2018). Ten units intravenous oxytocin over 2-4 h is as effective as 30 units over 8-12 h in preventing postpartum hemorrhage after cesarean section: A randomized controlled trial. Indian Journal of Pharmacology, 50(5), 279-283. https://doi.org/10.4103/ijp.IJP_419_18

Colucci, G., Helsing, K., Biasiutti, F. D., Raio, L., Schmid, P., Tsakiris, D. A., Eberle, B., Surbek, D., Lämmle, B., \& Alberio, L. (2018). Standardized management protocol in severe postpartum hemorrhage: A single-center study. Clinical and Applied Thrombosis/Hemostasis: Official Journal of the International Academy of Clinical and Applied Thrombosis/Hemostasis, 24(6), 884-893. https://doi.org/10.1177/1076029618758956

Ducloy-Bouthors, A. S., Mercier, F. J., Grouin, J. M., Bayoumeu, F., Corouge, J., Le Gouez, A., Rackelboom, T., Broisin, F., Vial, F., Luzi, A., Capronnier, O., Huissoud, C., Mignon, A., \& FIDEL working group. (2021). Early and systematic administration of fibrinogen concentrate in postpartum haemorrhage following vaginal delivery: The FIDEL randomised controlled trial. BJOG: An International Journal of Obstetrics and Gynaecology, 128(11), 1814-1823. https://doi.org/10.1111/1471-0528.16699

Dumont, A., Bodin, C., Hounkpatin, B., Popowski, T., Traoré, M., Perrin, R., \& Rozenberg, P. (2017). Uterine balloon tamponade as an adjunct to misoprostol for the treatment of uncontrolled postpartum haemorrhage: A randomised controlled trial in Benin and Mali. BMJ Open, 7(9), e016590. https://doi.org/10.1136/bmjopen-2017-016590

Durocher, J., Dzuba, I. G., Carroli, G., Morales, E. M., Aguirre, J. D., Martin, R., Esquivel, J., Carroli, B., \& Winikoff, B. (2019). Does route matter? Impact of route of oxytocin administration on postpartum bleeding: A double-blind, randomized controlled trial. PLOS ONE, 14(10), e0222981. https://doi.org/10.1371/journal.pone.0222981

Koch, D. M., \& Rattmann, Y. D. (2019). Uso do misoprostol no tratamento da hemorragia pós-parto: Uma abordagem farmacoepidemiológica. Einstein (São Paulo), 18, 1-7. https://doi.org/10.31744/einstein_journal/2020AO5029

Li, B., Miners, A., Shakur, H., Roberts, I., \& WOMAN Trial Collaborators. (2018). Tranexamic acid for treatment of women with post-partum haemorrhage in Nigeria and Pakistan: A cost-effectiveness analysis of data from the WOMAN trial. The Lancet. Global Health, 6(2), e222-e228. https://doi.org/10.1016/S2214-109X(17)30467-9

OPAS. (2018). Recomendações assistenciais para prevenção, diagnóstico e tratamento da Hemorragia Obstétrica. OPAS.

Shady, N. W., Sallam, H. F., Elsayed, A. H., Abdelkader, A. M., Ali, S. S., Alanwar, A., \& Abbas, A. M. (2019). The effect of prophylactic oral tranexamic acid plus buccal misoprostol on blood loss after vaginal delivery: A randomized controlled trial. The Journal of Maternal-Fetal \& Neonatal Medicine: The Official Journal of the European Association of Perinatal Medicine, the Federation of Asia and Oceania Perinatal Societies, the International Society of Perinatal Obstetricians, 32(11), 1806-1812. https://doi.org/10.1080/14767058.2017.1418316

Sweed, M., El-Said, M., Abou-Gamrah, A., \& Ali, M. (2019). Comparison between 200, 400 and 600 microgram rectal misoprostol before cesarian section: A randomized clinical trial. The Journal of Obstetrics and Gynaecology Research, 45(3), 585-591. https://doi.org/10.1111/jog.13883

WOMAN Trial Collaborators. (2017). Effect of early tranexamic acid administration on mortality, hysterectomy, and other morbidities in women with postpartum haemorrhage (WOMAN): An international, randomised, double-blind, placebo-controlled trial. Lancet (London, England), 389(10084), 2105-2116. https://doi.org/10.1016/S0140-6736(17)30638-4

Zargar, M., Nikbakht, R., \& Ahmadi, M. (2018). The effect of tranexamic acid on preventing post-partum hemorrhage due to uterine atony: A triple-blind Randomized Clinical Trial. Current Clinical Pharmacology, 13(2), 136-139. https://doi.org/10.2174/1574884713666180507101002 